\title{
Colonização e espécies de Candida em pacientes submetidos à radioterapia cervicofacial
}

\section{Candida colonization and species in patients submitted to head and neck radiotherapy}

Paulo Rogério Ferreti Bonan'; Fábio Ramoa Pires²; Márcio Ajudarte Lopes³; Osvaldo Di Hipólito Júnior ${ }^{3}$

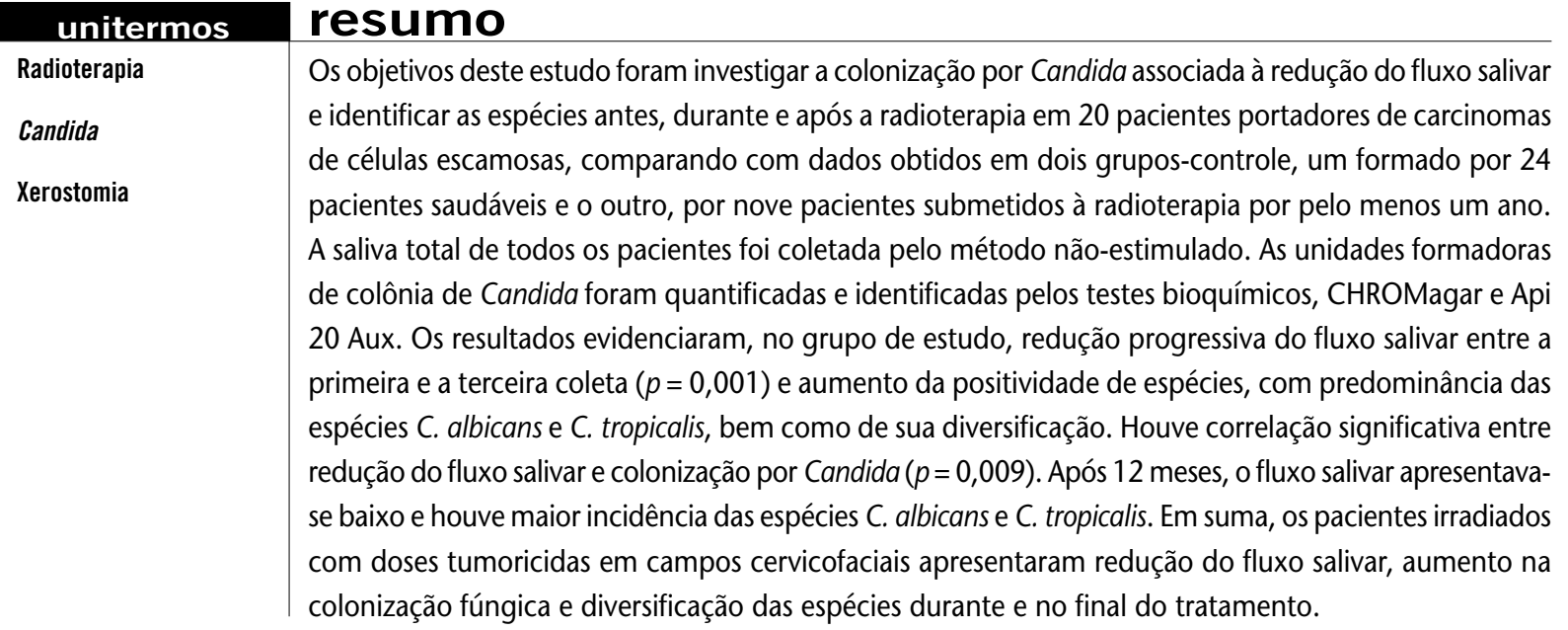

abstract

The aims of this study were to investigate Candida carriage associated with reduced salivary flow and to identify Candida species in 20 patients with squamous cell carcinoma before, during and after head and neck radiotherapy, comparing with 2 control groups, one compounded by 24 healthful patients and the other, by nine patients submitted to head and neck radiotherapy one year at least. Whole non stimulated saliva were collected from all patients. Candida unit form colonies were quantified and identified using biochemistry tests, CHROMagar and API 20 Aux. The results showed progressive reduction of salivary flow during radiotherapy $(\mathrm{p}=0.001)$ and increased Candida carriage, mainly $\mathrm{C}$. tropicalis and $\mathrm{C}$. albicans, with species diversification. The correlation between reduced salivary flow and increased Candida carriage was statistically demonstrated ( $\mathrm{p}=0.009$ ). After 12 months of the radiotherapy, the patients showed higher incidence of $\mathrm{C}$. albicans and $\mathrm{C}$. tropicalis. In conclusion, head and neck irradiated patients showed reduced salivary flow during radiotherapy, increasing of Candida colonization and higher variety of Candida species on saliva than non irradiated patients during and after radiotherapy. key words

Radiotherapy

Candida

Xerostomy 


\section{Introdução}

A radioterapia de fonte externa $(\mathrm{RxT})$ é uma das modalidades terapêuticas mais empregadas no tratamento do câncer em cabeça e pescoço, isoladamente ou associada a cirurgia e/ou quimioterapia( ${ }^{(3)}$. Embora os benefícios da radiação tumoral sejam evidentes, efeitos colaterais resultantes da radioterapia em campos cervicofaciais incluem alterações em superfície mucosa, ossos, glândulas salivares e dentes ${ }^{(11,28)}$.

As glândulas salivares expostas à radiação demonstram alterações como atrofia e degeneração da porção secretora, levando à redução no fluxo salivar e, conseqüentemente, à xerostomia, queixa principal dos pacientes irradiados em cabeça e pescoço ${ }^{(9,14)}$. Normalmente, após 100 cGy, o que corresponde à segunda semana de tratamento radioterápico, o fluxo salivar reduz significativamente e se torna viscoso, com modificações no pH e nos níveis de íons e imunoglobulinas ${ }^{(23)}$. Esses pacientes apresentam fibrose e risco aumentado de cáries e infecções, sendo candidose a

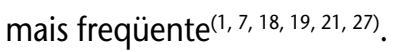

O aumento da colonização fúngica na cavidade bucal e suas manifestações clínicas estão diretamente associados a redução quantitativa e alterações qualitativas da saliva ${ }^{10,}$ 25, 26). Em adição, idade, uso do tabaco, imunossupressão, higiene bucal deficiente e antibioticoterapia contribuem para o aumento da colonização de Candida em pacientes irradiados em cabeça e pescoço ${ }^{(15,22,26)}$. Durante o curso radioterápico, foi demonstrada a diversificação de espécies de Candida, bem como o aumento da incidência da colonização de espécies como Candida albicans e Candida tropicalis ${ }^{(17,20)}$. Mesmo após a RxT, com a continuidade da xerostomia, a quantificação de espécies de Candida pode ser alta ${ }^{(5)}$. Ainda que as relações supracitadas sejam bem conhecidas, poucos estudos analisam diretamente a colonização de Candida em pacientes irradiados em cabeça e pescoço durante o curso radioterápico ${ }^{(13,17,20)}$. Portanto, os objetivos deste estudo são relacionar o fluxo salivar com a colonização fúngica antes, durante e após a radioterapia, verificar a diversificação de espécies e comparar com dados obtidos de pacientes submetidos à RxT há pelo menos um ano e com os de pacientes saudáveis.

\section{Materiais e métodos}

O presente estudo foi realizado na Faculdade de Odontologia de Piracicaba da Universidade Estadual de Campinas (FOP/UNICAMP) e no Hospital dos Fornecedores de Cana de Piracicaba, onde foram avaliados 20 pacientes portadores de neoplasias malignas em cabeça e pescoço com indicação para a realização de RxT em campos cervicofaciais (grupo I). Esses pacientes foram submetidos a RxT em doses tumoricidas, cirurgia e quimioterapia não concomitantes, não influenciando na coleta das amostras. Como grupos de controle foram selecionados aleatoriamente 24 pacientes com boa saúde e sem desordens ou processos patológicos que poderiam levar à xerostomia (grupo II). Um outro grupo (grupo III) foi formado por nove pacientes, os quais foram submetidos à RxT em doses tumoricidas em campos cervicofaciais após pelo menos um ano de realização devido a neoplasias malignas em cabeça e pescoço.

A coleta não-estimulada de saliva foi realizada em todos os pacientes por 5 minutos, depositando-se diretamente seu conteúdo líquido produzido nesse intervalo em um frasco estéril. No grupo I, as coletas foram realizadas antes do início da RxT, entre a 15a e 23a sessão e após o tratamento. Nos grupos II e III foi realizada coleta salivar única no momento da consulta. As amostras de saliva foram pesadas, convertidas para a unidade $\mathrm{ml} / \mathrm{min}$, homogeneizadas e diluídas em solução salina estéril. Alíquotas de 0,1 ml de amostras de saliva puras e diluídas a $10^{-1}$ em solução fisiológica estéril foram semeadas em placas de Petri, em duplicata, e distribuídas de forma homogênea com alça de Drigalsky, contendo o meio ágar Sabouraud-dextrose (Difco, B\&D, Brasil) com cloranfenicol a 1\% (Quimicetina ${ }^{\circledR P f i z e r, ~ B r a s i l) . ~}$ As placas semeadas em duplicata, duas para a amostra pura e duas para a diluição, foram encubadas em estufa a $37^{\circ} \mathrm{C}$ e lidas após 48 horas. A média das unidades formadoras de colônia (CFU) foi obtida. A partir da contagem de CFU/ml de saliva os pacientes foram classificados em negativos $(\mathrm{CFU} / \mathrm{ml}=0)$, portadores (CFU $/ \mathrm{ml}<400$ ) e positivos (CFU/ $\mathrm{ml}>400)$, como proposto por Olsen et al.(16). Quando as CFUs apresentavam diferenças morfológicas de tamanho (colônias grandes e pequenas) e superfície (lisa ou rugosa), foram isoladas separadamente para identificação.

Espécies de Candida foram identificadas pelos testes de formação do tubo germinativo e do clamidosconídeo, em que a positividade para ambos identificava a espécie $C$. albicans. Quando da negatividade para C. albicans, testes de fermentação e assimilação foram realizados seguindo-se a metodologia proposta por Sandvén ${ }^{(24)}$. Em adição, em casos de dúvida investigativa, processou-se a identificação por utilização do meio cromogênico CHROMagar Candida (CHROMagar Company, França), que diferenciou pela coloração as espécies $C$. albicans e C.tropicalis de outras espécies, e do teste de identificação API 20 AUX (BioMérieux Company, 
França) para identificar espécies não-albicans. Esse estudo foi aprovado pelo comitê de ética da FOP/UNICAMP e os pacientes consentiram os procedimentos de coleta. As análises estatísticas foram realizadas utilizando-se o software SPSS (Chicago, IL, USA), os testes não-paramétricos de Mann-Whitney, Kruskal-Wallis e a correlação de Spearman bicaudada, com o nível de significância de $5 \%$.

\section{Resultados}

A média etária dos pacientes do grupo I foi de 59,6 anos ( $\pm 10,08$ anos), sendo formado por 17 homens (85\%) e três mulheres (15\%). Quanto ao grupo II, a idade média foi de 58,4 anos ( $\pm 11,20$ anos), sendo composto por 19 homens $(79,1 \%)$ e cinco mulheres (20,9\%). No grupo III, a idade média foi de 56,8 anos $( \pm 13,9)$, sendo oito homens (89\%) e uma mulher (11\%).

O grupo I apresentou todos os pacientes com carcinomas de células escamosas, sendo cinco (25\%) em assoalho bucal, cinco (25\%) em orofaringe, quatro (20\%) em língua, dois (10\%) em mucosa jugal e quatro (20\%) em outras localizações. O estádio clínico (tumor/linfonodo/metástase [TNM]) foi dividido em IV (12 casos, 60\%), III (3 casos, 15\%), I (3 casos, 15\%) e II (2 casos, 10\%). A dose total média de radiação em campos cervicofaciais foi de 7.070 cGy, variando de 6.000 cGy a 8.260 cGy. Em todos os pacientes, os campos de radiação envolveram parótidas, glândulas submandibulares e muitas glândulas salivares menores. $\mathrm{O}$ valor médio do fluxo salivar obtido nos pacientes do grupo I foi de $0,5962 \mathrm{ml} / \mathrm{min}$ na primeira coleta, $0,23247 \mathrm{ml} / \mathrm{min}$ na segunda coleta e $0,22012 \mathrm{ml} / \mathrm{min}$ na terceira coleta. O fluxo salivar obtido do grupo II foi, em média, 0,6736 $\mathrm{ml} / \mathrm{min}$, e do grupo III, 0,29022 ml/min. Na coleta inicial, não houve diferença estatisticamente significativa entre os grupos I e II $(p=0,654)$. Contudo as médias de fluxo salivar na segunda e terceira coletas e do grupo III foram estatisticamente diferentes da coleta inicial do grupo I $(p=0,001, p=0,001, p=$ 0,026 , respectivamente). A distribuição dos valores de fluxo salivar intergrupos e no grupo I está expressa na Figura. A freqüência de isolamento de Candida e sua relação com o avanço do tratamento radioterápico estão demonstradas na Tabela 1. A condição de positividade para Candida se tornou mais evidente na metade do tratamento radioterápico e após um ano do fim do tratamento. Referente à associação entre a redução do fluxo salivar e o aumento da colonização fúngica houve relação negativa significativa pelo teste de correlação de Spearman bicaudado $(-269, p=0,009)$. A correlação significativa evidenciou a relação entre o aumento da colonização fúngica e a redução do fluxo salivar. No tocante à diversificação de espécies, obtidas das amostras isoladas por diferença morfológica, na primeira coleta do grupo I, 23 espécies foram encontradas; na segunda, 29 espécies; e na terceira, 27. No grupo II, 23 espécies foram encontradas e no grupo III, 12. As espécies encontradas e sua freqüência estão listadas na Tabela 2. Observaram-se maior prevalência das espécies $C$. tropicalis e $C$. albicans e diversificação de espécies durante e no término da RxT. Quanto à utilização dos métodos de identificação, o bioquímico e o CHROMagar identificaram a maioria das espécies, ao passo que o API 20 Aux foi utilizado no reconhecimento de espécies que não foram identificadas pelos métodos anteriores. Os métodos expostos não identificaram amostras de $C$. dubliniensis. As espécies cuja caracterização não foi conseguida por nenhum dos métodos foram denominadas Candida sp.

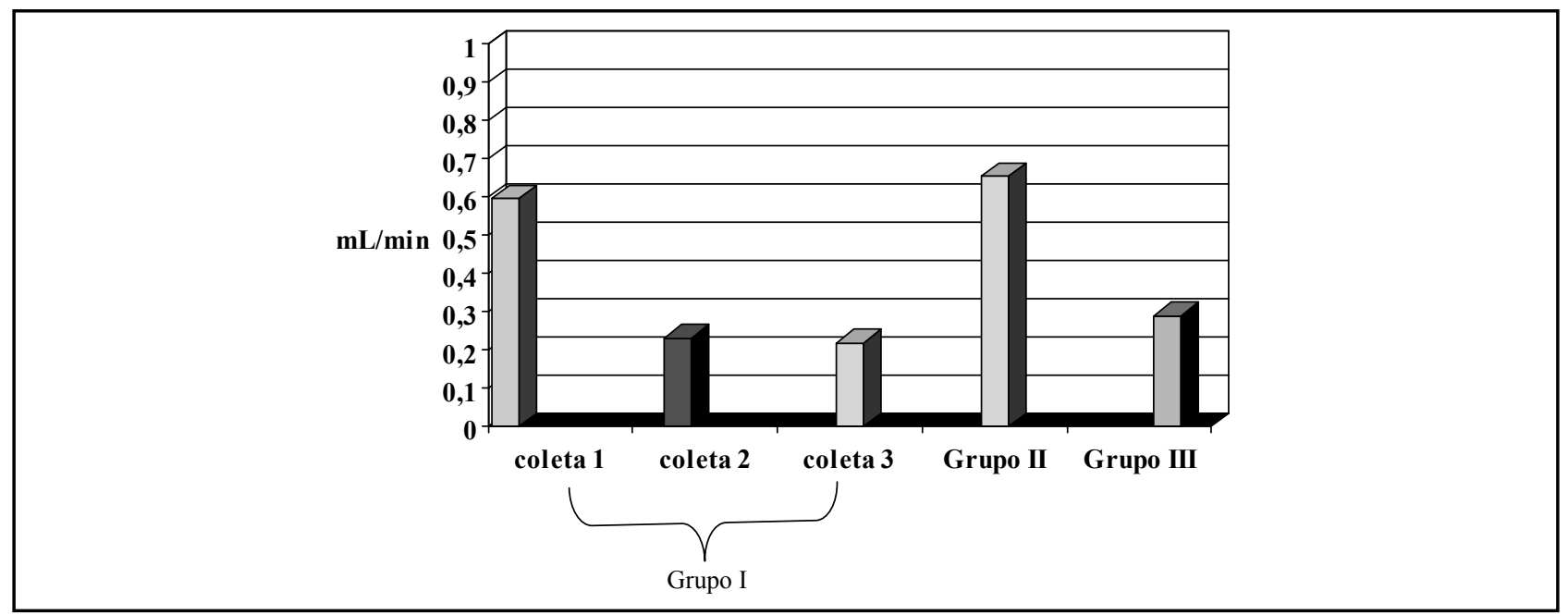

Figura - Distribuição do fluxo salivar nos grupos I, II e III a partir de amostras obtidas em coletas isoladas não estimuladas (grupos II e III) e coletas no início, meio e fim do tratamento radioterápico (grupo I) 
Distribuição dos pacientes quanto à condição de positividade para Candida (frequiência de isolamento) nas três coletas e nos grupos I e II $(n=53)$ relacionada com a cultura do microorganismo a partir de

Tabela 1 saliva coletada e semeada em meio de cultura ágar Sabouraud-dextrose com cloranfenicol a $1 \%$

\begin{tabular}{lccc}
\hline Grupo & Condição & \\
Grupo I & Pogativo $(\%)$ & $3(15)$ & Positivo (\%) \\
Primeira coleta $(n=20)$ & $3(15)$ & $3(15)$ & $14(70)$ \\
Segunda coleta $(n=20)$ & $1(5)$ & $3(15,8)$ & $16(80)$ \\
Terceira coleta $(n=19)^{*}$ & $2(10,5)$ & $6(25)$ & $14(73,7)$ \\
Grupo II $(n=24)$ & $6(25)$ & $1(11)$ & $12(50)$ \\
Grupo III $(n=9)$ & $0(0)$ & $8(89)$ \\
\hline
\end{tabular}

*Uma amostra não pôde ser processada.

Distribuição percentual das espécies de Candida encontradas nas coletas dos grupos I, II e III a partir Tabela 2 do isolamento prévio em meio de cultura e diversificação das espécies durante as coletas no grupo I

\begin{tabular}{lccccc}
\hline Espécies & \multicolumn{3}{c}{ Grupo I } & Grupo II & Grupo III \\
Coleta & Coleta 1 & Coleta 2 & Coleta 3 & & \\
C. albicans (\%) & 70 & 39,5 & 37 & 61 & 41,6 \\
C. tropicalis (\%) & 33,5 & 50 & 44,5 & 26 & 50 \\
C. krusei (\%) & - & - & 3,7 & 4,3 & - \\
C. guilhermondii (\%) & - & - & - & 4,3 & - \\
C. humicula (\%) & - & 3,5 & - & - & - \\
C. parapsilosis (\%) & - & 3,5 & 7,4 & - & - \\
C. lusitanae (\%) & - & - & 3,7 & - & - \\
Candida sp. (\%) & - & 3,5 & 3,7 & 4,3 & 8,4 \\
\hline
\end{tabular}

\section{Discussão}

A hipossalivação é um dos efeitos colaterais mais freqüentes associados ao tratamento radioterápico em campos cervicofaciais ${ }^{(23,27)}$. A redução do fluxo está relacionada com o aumento da colonização oral por Candida, bem como a microbiota bacteriana acidogênica e acidúrica, responsável pelo desenvolvimento de cáries dentárias $(11,12,18,19,25,27)$.

O fluxo salivar durante a radioterapia apresentou redução significativa progressiva durante e após a RxT, em comparação com a coleta inicial e com pacientes saudáveis. O fluxo salivar permaneceu reduzido mesmo um ano depois do tratamento radioterápico ${ }^{(12)}$. Em um estudo de avaliação similar do fluxo salivar, comprovamos a redução do fluxo salivar dos pacientes irradiados e pós-RxT ${ }^{(4)}$. Em adição, a relação entre a redução do fluxo salivar e o incremento fúngico foi demonstrada estatisticamente. Em um estudo com metodologia similar, Ramirez-Amador et al. ${ }^{(19)}$ associa- ram estatisticamente a correlação entre a redução do fluxo salivar e o aumento da colonização fúngica.

O aumento na colonização fúngica é propiciado por, além de redução do fluxo salivar, higiene oral deficiente,

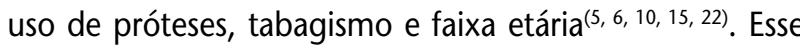
fato explica a razão da positividade maior para Candida em pacientes pré-RxT em comparação com pacientes saudáveis. Reconhecidamente, esses pacientes apresentam higiene oral deficitária, muitas vezes dificultada por tumores volumosos, evidenciada pela predominância do estádio avançado encontrado e pelo perfil tabagista crônico, que também é contributivo para a colonização fúngica. Um estudo que avaliou comparativamente a colonização por Candida durante a RxT e a sua presença antes do tratamento evidenciou que $56 \%$ dos pacientes tinham Candida e que 30\% das espécies foram classificadas como C. albicans ${ }^{(17)}$. Em outro estudo realizado por Reeding et al. ${ }^{(20)} \mathrm{em}$ pacientes irradiados, foram isoladas espécies de Candida em 51\% dos pacientes-controle e em 
$73 \%$ dos pacientes estudados, com prevalência maior de C. albicans (78\%) em lesões fúngicas. Embora difiram na metodologia, nesse estudo encontramos que a maioria dos pacientes foi positiva para Candida (70\%) e que a espécie C. albicans foi a mais freqüentemente encontrada no início do tratamento (70\%). O grupo II, composto por pacientes saudáveis, apresentou perfil etário muito semelhante ao do grupo I, o que minorou a sua influência na diferença de quantificação intergrupos. No grupo III, $89 \%$ dos pacientes foram positivos para Candida, apresentando fluxo salivar reduzido, o que contribuiu para a colonização, embora um estudo afirme que ocorram alterações na microbiota oral com redução da colonização fúngica nesse período ${ }^{(12)}$.

$\mathrm{O}$ aumento na colonização fúngica durante a RxT foi demonstrado por Paula et al. ${ }^{(17)}$, que mostraram aumento da colonização progressiva de Candida (72\% em comparação com $56 \%$ prévios a RxT) durante o tratamento associado principalmente a C. albicans e C. tropicalis (36\% e 16\%, respectivamente). De forma similar, Ramirez-Amador et al.(19) demonstraram alta positividade para C. albicans durante o tratamento e no seu término $(85 \%$ e $68 \%$, res- pectivamente). A explicação mais plausível para a redução do percentual de incidência de C. albicans tem relação com o aumento da positividade para espécies não-albicans, como demonstrado neste estudo, com a inversão da predominância para a C. tropicalis( ${ }^{(8)}$. A diversificação entre espécies durante e após a realização da RxT foi constatada por estudos prévios ${ }^{(8,17)}$. Contudo, as espécies C. albicans e C. tropicalis, conhecidas por sua patogenicidade, em todos os estudos, assim como nesta avaliação, foram as mais freqüentemente encontradas $(13,17,20)$,

\section{Conclusões}

Em suma, os pacientes a serem submetidos ao tratamento radioterápico em cabeça e pescoço apresentam alta positividade para Candida antes do tratamento radioterápico. Além disso, com o aumento da xerostomia, cresceu a colonização fúngica durante a RxT, com predominância das espécies $C$.albicans e $C$. tropicalis e diversificação das espécies encontradas.

\section{Referências}

1. ALMSTAHL, A.; WISKTROM, M. Oral microflora in subjects with reduced salivary secretion. J Dent Res, v. 78, p. 1410-6, 1999.

2. BELAZI, M. et al. Oral Candida isolates in patients undergoing radiotherapy for head and neck cancer: prevalence, azole susceptibility profiles and response to antifungal treatment. Oral Microbiol Immunol, v. 19, p. 347-51, 2004.

3. BLOZIS, G. G.; ROBINSON, J. E. Oral tissues changes caused by radiation therapy and their management. Dent Clin North Am, s/n, p. 643-56, 1968.

4. BONAN, P. R et al. Evaluation of salivary flow in patients during head and neck radiotherapy. Pesqui Odontol Bras, v. 17, p. 156-60, 2003.

5. BUDTZ-JÖRGENSEN, E. The significance of Candida albicans in denture stomatitis. Scand J Dent Res, v. 82, p. 151-90, 1974.

6. BUDTZ-JÖRGENSEN, E.; STENDERUP, A.; GRABOWSKY, $M$. An epidemiological study of yeast in elderly denture wearers. Community Dent Oral Epidemiol, v. 3, p. 1159, 1975.

7. CARL, W. Local radiation and systemic chemotherapy: preventing and managing the oral complications. J Am Dent Assoc, v. 124, p. 119-23, 1993.

8. DAHIYA, M. C. et al. Oropharyngeal candidiasis caused by non-albicans yeast in patients receiving external beam radiotherapy for head-and-neck cancer. Int J Radiat Oncol Biol Phys, v. 57, p. 79-83, 2003.

9. EPSTEIN, J. B. et al. Quality of life and oral function following radiotherapy for head and neck cancer. Head Neck, v. 21, p. 1-11, 1999.

10. EPSTEIN, J. B.; FREILICH, M. M.; LE, N. D. Risk factors for oropharyngeal candidiasis in patients who receive radiation therapy for malignant conditions of the head and neck. Oral Surg Oral Med Oral Pathol, v. 76, p. 167-74, 1993

11. GARG, A. K.; MALO, M. Manifestations and treatment of xerostomia and associated oral effects secondary to head and neck radiation therapy. J Am Dent Assoc, v. 128, p. 1128-33, 1997

12. GROTZ, K. A. Long-term oral Candida colonization, mucositis and salivary function after head and neck radiotherapy. Support Care Cancer, v. 11, p. 717-21, 2003.

13. LEUNG, W. K. et al. Oral colonization, phenotypic and genotypic profiles of Candida species in irradiated, dentate, xerostomic, nasopharyngeal carcinoma survivors. J Clin Microbiol, v. 38, p. 2219-26, 2000.

14. LOPES, M. A. et al. Reconhecendo e controlando os efeitos colaterais da radioterapia. Revista da $A P C D$, v. 241-4, 1998.

15. LOPEZ-JORNET, M. P.; BERMEJO-FENOLL, A. Is there an aged-dependent decrease in resting secretion of saliva 
of healthy person? A study of 1493 subjects. Braz Dent J, v. 5, p. 93-8, 1994.

16. OLSEN, I.; STENDERUP, A. Clinical-mycologic diagnosis of oral yeast infections. Acta Odontol Scand, v.48, p. 11-8, 1990.

17. PAULA, C. R.; SAMPAIO, M. C. C.; BIRMAN, E. G.; SIQUEIRA, A. M. Oral yeasts in patients with cancer of the mouth, before and during radiotherapy. Mycopathologia, v. 112, p. 119-24, 1990.

18. QUINDOS, G. et al. Utility of fluoroplate Candida for the rapid identification of Candida albicans. Enferm Infecc Microbiol Clin, v. 14, p. 586-9, 1996.

19. RAMIREZ-AMADOR, V. et al. Candidal colonization and oral candidiasis in patient undergoing oral and pharyngeal radiation therapy. Oral Surg Oral Med Oral Pathol Oral Radiol Endod, v. 84, p. 149-53, 1997.

20. REDDING, S.W. et al. Epidemiology of oropharyngeal Candida colonization and infection in patients receiving radiation for head and neck cancer. J Clin Microbiol, v. 37, p. 3896-900, 1999.

21. REGELINK, G. et al. Efficacy of a synthetic polymer saliva substitute in reducing oral complaints of patients suffering from irradiation-induced xerostomia.
Quintessense Int, v. 29, p. 383-8, 1998.

22. ROSSIE, K. M. et al. Influence of radiation therapy on oral Candida albicans colonization: a quantitative assessment. Oral Surg Oral Med Oral Pathol, v. 64, p. 698-701, 1987.

23. ROTHWELL, B. R. Prevention and treatment of orofacial complications of radiotherapy. J Am Dent Assoc, v. 114, p. 316-22, 1987.

24. SANDVEN, P. Laboratory identification and sensitivity testing of yeast isolates. Acta Odontol Scand, v. 48, p. 27-36, 1990.

25.SCULLY, C.; EPSTEIN, J. B. Oral health care for the cancer patient. Eur J Cancer B Oral Oncol, v. 32B, p. 281-92, 1996.

26. TARTARO, G. P.; ITRO, A.; GRISOLIA, G. Oral candidiasis following radiotherapy for neoplasms of oromaxillofacial area. Minerva Stomatol, v. 45, p. 451-4, 1996.

27. WEERKAMP, A. H. et al. Effect of the application of mucinbased saliva substitute on oral microflora of xerostomic patients. J Oral Pathol, v. 16, p. 474-8, 1998.

28. WHITMYER, C. C.; ESPOSITO, S. J.; TEREZHALMY, G. T. Radiotherapy for head and neck neoplasm. Gen Den, v. 45, p. 363-70, 1997. 